\title{
A QUALIDADE DA COPA DO MUNDO DA FIFA BRASIL 2014 NAS CIDADES-SEDE
}

THE QUALITY OF THE 2014 FIFA WORLD CUP BRAZIL IN THE HOST CITIES

\section{LA CALIDAD DE LA COPA MUNDIAL DE LA FIFA BRASIL 2014 EN CIUDADES SEDE}

Thiago de Oliveira Santos*, Abel Correia *, Rui Biscaia** Carla Araújo***, Carlos Augusto Mulatinho de Queiroz Pedroso ${ }^{\star \star * \star}$ Fábio Mucio Stinghen ${ }^{\star \star * * *}$,

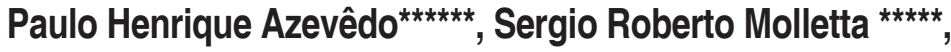
Varley Teoldo Costa ${ }^{\star * * * * *}$ Vilde Gomes de Menezes ***

\section{Palavras-chave}

Futebol.

Cidades.

Percepção

Gestão da

qualidade.

Keywords

Soccer.

Cities.

Perception.

Quality

management.

Palabras clave

Fútbol.

Ciudades.

Percepción.

Gestión de calidad.
Resumo: $O$ objetivo deste estudo foi verificar se a percepção que os torcedores têm da qualidade da Copa do Mundo da FIFA Brasil 2014 varia consoante os locais onde o evento foi realizado. Foram recolhidos 3.042 questionários em cinco cidades-sede da Copa. Os resultados obtidos através de uma análise fatorial confirmatória indicaram boas qualidades psicométricas do modelo usado para medir a qualidade. Os testes MANOVA indicaram diferenças significativas na avaliação da qualidade do evento entre os participantes nas cinco cidades-sede. Os resultados foram discutidos, apontando-se as principais implicações na organização de eventos esportivos, bem como sugestões para futuras pesquisas.

Abstract: The purpose of this study was to verify whether the perception of quality of the 2014 FIFA World Cup Brazil varies depending on where the event was hosted. A total of 3,042 questionnaires were collected in five cities hosting the World Cup. Results from confirmatory factor analysis indicated good psychometric qualities of the model used to measure quality. Through MANOVA tests, significant differences in the event quality were observed among participants from five host cities. The results were discussed and managerial implications and suggestions for future studies were provided.

Resumen: El objetivo de este estudio fue determinar si la percepción que tiene la afición sobre la calidad de la Copa Mundial de la FIFA Brasil 2014, varía de acuerdo a las ciudades donde el evento fue realizado. Se recogieron 3.042 cuestionarios en cinco ciudades sede. Los resultados obtenidos mediante un análisis factorial confirmatorio indicaran buenas cualidades psicométricas del modelo utilizado para medir la calidad del evento. Los testes MANOVA indicaron diferencias significativas en la evaluación de la calidad del evento entre los participantes en las cinco ciudades. Los resultados han sido discutidos, señalando las principales implicaciones en la organización de eventos deportivos, así como sugerencias para futuras investigaciones..
* Universidade de Lisboa. Lisboa, Portugal.

E-mail: thiago_os@hotmail.com

**Universidade Europeia. Universidade de Lisboa. Lisboa, Portugal. E-mail: rbiscaia@fmh.ulisboa.pt

${ }^{* \star *}$ Universidade Salgado de Oliveira. Rio de Janeiro, RJ, Brasil. E-mail: carla_mpompilho@hotmail.com,

****Universidade de Pernambuco. Recife, PE, Brasil. E-mail: carlosaugustomulatinho@gmail.com

*****Universidade Tecnológica Federal do Paraná. Curitiba, PR, Brasil. E-mail: fabiosting@gmail.com

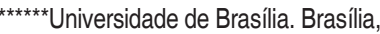
DF, Brasil. E-mail: pha@gesporte.net

****Universidade Federal de Minas Gerais. Belo Horizonte, MG, Brasil. E-mail: vtcosta@ hotmail.com

Recebido em: 10-07-2015 Aprovado em: 20-12-2015

(c) (1) (8) Licence 


\section{INTRODUÇÃO}

Acolher um megaevento esportivo pode impactar diretamente na qualidade de vida dos residentes (TAKS; CHALIP; GREEN, 2015). O número significativo de espectadores que assistem aos megaeventos e a exposição mediática podem projetar mundialmente a imagem dos anfitriões (DEMBEK; WLOCK, 2014). Para sediar um evento como a Copa do Mundo da FIFA os custos são elevados, principalmente se for necessário investir na construção e remodelação de infraestruturas esportivas e/ou urbanas (SANT; MASON, 2015). Por exemplo, a África do Sul investiu cerca de R\$ 12 bilhões no mundial em 2010. Por sua vez, o Brasil investiu cerca de R \$ 24 bilhões para sediar a Copa do Mundo de 2014 (FORBES, 2014), enquanto a Rússia pretende investir R\$ 40 bilhões para sediar o mundial em 2018 (FÉDÉRATION INTERNATIONALE DE FOOTBALL ASSOCIATION, 2013).

Apesar dos altos custos, os impactos econômicos e sociais (e.g., geração de emprego e renda, atração de capital estrangeiro, desenvolvimento esportivo, aumento no turismo e aceleração do desenvolvimento urbano) gerados pelos megaeventos como a Copa do Mundo da FIFA (KAPLANIDOU, 2012) são referenciados pelos organizadores como justificativa para a organização desses megaeventos (HEERE et al., 2013). Para Santos (2014), a realização da Copa do Mundo da FIFA em 2014 representa parte de uma estratégia mais ampla usada pelo governo brasileiro para melhorar as relações internacionais e demonstrar a vitalidade econômica, social e política do país.

A confirmação do Brasil como país-sede da Copa de 2014 iniciou uma disputa interna entre 22 cidades para 12 vagas como subsedes do mundial. Após avaliação do comitê organizador foram escolhidas: Belo Horizonte, Brasília, Cuiabá, Curitiba, Fortaleza, Manaus, Natal, Porto Alegre, Recife, Rio de Janeiro, Salvador e São Paulo. Dessas, onze são capitais estaduais, além de Brasília. Todas são importantes cidades com indicadores sociais e características culturais bastante distintas (RODRIGUES SOARES, 2013). O contexto social e cultural tem sido apontado na literatura como sendo importante na avaliação da qualidade dos eventos (AU, 1999, AGARWAL; MALHORTA; BOLTON, 2010). Em linha com esta ideia, estudos têm demonstrado a importância de se identificar fatores que influenciam a percepção da qualidade do evento para os consumidores (PORTER; FLETCHER, 2008, INOUE; HAVARD, 2014).

A percepção do consumidor da qualidade do serviço tem recebido atenção dos pesquisadores devido à criação de benefícios para as organizações (KOO et al., 2009, THEODORAKIS et al., 2013). Zeithaml e Bitner (2003) referem-se à qualidade do serviço como uma atitude geral ou julgamento sobre a superioridade de um serviço. No contexto dos eventos esportivos a qualidade do serviço é definida como a avaliação do consumidor sobre a excelência do serviço prestado pelo anfitrião (LIU; TAYLOR; SHIBLI, 2009). Diversas escalas têm sido propostas para avaliar a percepção dos consumidores sobre a qualidade dos eventos (e.g., BISCAIA et al., 2013, THEODORAKIS, ALEXANDRIS, 2008, WAKEFIELD; BLODGETT; SLOAN, 1996, YOSHIDA; JAMES, 2011). Recentemente, a escala proposta por Biscaia et al. (2013), composta por dez dimensões relacionadas com os atributos técnicos, funcionais e estéticos do serviço, mostrou boas qualidades psicométricas quando testada entre torcedores de uma liga profissional de futebol, constituindo-se como um bom instrumento para avaliar a qualidade dos eventos esportivos. Ainda assim, pouco se sabe sobre a qualidade dos megaeventos esportivos que são realizados de forma menos regular e em diversas cidades em simultâneo (e.g., subsedes 
da Copa do Mundo). Florek, Breitbarth e Conejo (2008), num estudo longitudinal realizado na Copa do Mundo da FIFA da Alemanha 2006, utilizando medidas para avaliar a percepção de neozelandeses sobre a qualidade do evento (e.g., segurança, atmosfera do evento, experiência com os torcedores e infraestruturas), concluiram que o fato destes experienciarem o evento num contexto cultural e social diferente tem impacto direto na avaliação da qualidade. Outros estudos (CHAO et al., 2012, JEEYOON; JOON HO; YU-KYOUM, 2014) sugerem que os contextos cultural, social e econômico podem influenciar a percepção dos consumidores em relação à qualidade dos eventos. Neste sentido, o objetivo deste estudo foi verificar se a percepção da qualidade da Copa do Mundo da FIFA Brasil 2014 varia consoante as cidades-sede do evento.

\section{MÉTODO}

\subsection{Contexto do estudo}

Este estudo foi conduzido em cinco cidades-sede: Belo Horizonte, Brasília, Curitiba, Recife e Rio de Janeiro. Na Tabela 1 estão descritos indicadores que caracterizam cada uma das cidades.

Tabela 1 - Indicadores de desenvolvimento

\begin{tabular}{|c|c|c|c|c|c|c|c|c|}
\hline Subsede & População & $\begin{array}{c}\% \\
\text { PIB }^{1}\end{array}$ & IDHM $^{2}$ & $\begin{array}{l}\text { Rendi- } \\
\text { mento }^{3}\end{array}$ & $\begin{array}{c}\text { Pobreza } \\
(\%)^{4}\end{array}$ & Equipes $^{5}$ & $\begin{array}{l}\text { Público } \\
\text { médio }^{6}\end{array}$ & $\begin{array}{l}\text { Investimentos } \\
\text { para a Copa }\end{array}$ \\
\hline Belo Horizonte & 2.375 .151 & 1,37 & 0,810 & 1.226 & 0,7 & 2 & 22.205 & 2,6 \\
\hline Brasília & 2.570 .160 & 3,98 & 0,824 & 1.404 & 0,9 & 0 & - & 2,2 \\
\hline Curitiba & 1.751 .907 & 1,41 & 0,823 & 1.273 & 0,3 & 2 & 12.283 & 1,2 \\
\hline Recife & 1.537 .704 & 0,80 & 0,772 & 894 & 4,5 & 1 & 18.220 & 1,7 \\
\hline Rio de Janeiro & 6.320 .446 & 5,05 & 0,799 & 1.204 & 1,1 & 3 & 18.754 & 3,9 \\
\hline
\end{tabular}

${ }^{1}$ Participação no PIB do País; ${ }^{2}$ Índice de Desenvolvimento Humano Municipal: Iongevidade, educação e renda;

${ }^{3}$ Rendimento mensal domiciliar em $\mathrm{R} \$ ;{ }^{4}$ Rendimento mensal domiciliar de $\mathrm{R} \$ 70,00 ;{ }^{5}$ Equipes na Série $\mathrm{A}(2014) ;{ }^{6}$ Média de público na Série A (2014); ${ }^{7}$ Previsão $\mathrm{R} \$$ total em bilhões.

Fontes: IBGE, 2010; Portal da Transparência - Copa do Mundo 2014,

$<$ http://globoesporte.globo.com/futebol/brasileirao-serie-a/publico-brasileirao.html>. Autoria própria.

Estas cidades reúnem cerca de 14 milhões de habitantes e concentram 12,61\% do PIB nacional (INSTITUTO BRASILEIRO DE GEOGRAFIA E ESTATÍSTICA, 2010). Os residentes em Brasília têm o maior rendimento médio mensal ( $R$ \$ 1.404), enquanto os residentes em Recife têm o menor (R\$ 894) e cerca de $4 \%$ da população vive com menos de $R \$ 70,00$ por mês (INSTITUTO BRASILEIRO DE GEOGRAFIA E ESTATÍSTICA, 2010). No futebol, apenas Brasília não tem equipe na primeira divisão do campeonato brasileiro. Belo Horizonte, com duas equipes na série $A$, obteve a maior média de assistência aos jogos da competição em 2014. A estimativa de investimentos para a realização da Copa do Mundo nestas cinco cidades-sede foi em torno de $45 \%$ do valor total previsto de $\mathrm{R} \$ 27$ bilhões para os gastos com o torneio. Ainda assim, os valores em cada cidade variam. O Rio de Janeiro recebeu cerca de $\mathrm{R} \$ 3,8$ bilhões em investimentos, enquanto Curitiba pouco mais de $\mathrm{R} \$ 1$ bilhão.

\subsection{Medidas}

Para avaliar a percepção dos torcedores sobre a qualidade do evento foram adaptados os itens da escala proposta por Biscaia et al. (2013). Ainda que a qualidade de serviço seja 
tradicionalmente avaliada após o consumo (BISCAIA et al., 2013, CRONIN; BRADY, HULT, 2000), todos os itens foram adaptados para permitir aos participantes opinar sobre a qualidade do evento antes de entrar na arena. A decisão de recolher os dados antes dos jogos está relacionada com três aspectos. Primeiro, a FIFA permitiu a venda de bebidas alcoólicas dentro dos estádios durante a Copa do Mundo (CAETANO; PINSKY; LARANJEIRA, 2012), o que poderia comprometer as respostas dos participantes. Segundo, os resultados dos jogos tendem a afetar a percepção sobre as experiências de consumo (KOENIGSTORFER; GROEPPEL-KLEIN; KUNKEL, 2010), o que poderia levar os participantes a superestimarem 0 resultado do jogo na avaliação da qualidade do evento. Terceiro, tendo em conta as sugestões de cinco pesquisadores brasileiros, não foi considerado adequado recolher dados após os jogos, uma vez que as pessoas tendem a ir para casa rapidamente por questões de segurança ou a deslocar-se para as fan zones localizadas nas cidades em busca de mais celebrações (FÉDÉRATION INTERNATIONALE DE FOOTBALL ASSOCIATION, 2012). Além disso, é importante destacar que devido à dimensão temporal e à grande cobertura da mídia em torno deste tipo de megaevento, os torcedores tendem a construir sua percepção antes deste ocorrer. Para o efeito, referem-se às polêmicas que aconteceram no Brasil na preparação para a Copa (ESPN..., 2014).

Foram utilizados 24 itens relativos à qualidade do evento (BISCAIA et al., 2013) para avaliar os atributos: equipes, árbitros, pessoal de apoio, acessibilidades, segurança, design da arena, experiência com os torcedores e atmosfera do evento (três itens para cada atributo). Todos os itens do questionário foram medidos numa escala de Likert de sete pontos, variando entre "Discordo totalmente" (1) e "Concordo totalmente" (7).

Antes de aplicar o questionário, foi feita uma análise de conteúdo por parte de seis pesquisadores brasileiros de gestão do esporte, em termos de relevância e clareza dos itens para a população. Estes pesquisadores receberam um e-mail explicando o objetivo do estudo, os procedimentos de coleta dos dados, a descrição de cada dimensão e a lista de itens propostos. Os pesquisadores sugeriram a alteração da redação em 11 itens. As sugestões foram aceites e todos os itens foram colocados de forma aleatória num questionário, junto com questões sociodemográficas, para a realização de um pré-teste. Em seguida, 100 estudantes de cursos de Educação Física foram selecionados aleatoriamente em cinco universidades brasileiras das mesmas cidades onde a coleta dos dados foi planejada (20 alunos por instituição). Após 0 pré-teste, outros três itens foram reformulados. A versão final do questionário para medir a qualidade do evento foi então considerada adequada para a aplicação.

\subsection{PARTICIPANTES E COLETA DE DADOS}

Os dados foram coletados em 29 jogos: Belo Horizonte (6), Brasília (7), Curitiba (4), Recife (5), e Rio de Janeiro (7). Os procedimentos de coleta dos dados foram iguais nas cinco cidades-sede. Estabeleceu-se um supervisor e um grupo de inquiridores para cada cidade, sendo distribuídos no entorno das arenas. Os inquiridores abordaram potenciais participantes antes do início dos jogos, explicando o objetivo do estudo e solicitando a participação para responder ao questionário. Apenas indivíduos brasileiros ou residentes estrangeiros com domínio do português e com bilhete para entrar na arena foram selecionados para participar no estudo. Todos assinaram um termo de consentimento livre e esclarecido. Dos 5.000 
questionários distribuídos ( 1.000 em cada cidade-sede), 3.466 foram coletados. Após triagem, 3.042 respostas foram consideradas válidas para a análise. A amostra final incluiu 664 participantes de Belo Horizonte, 627 de Brasília, 522 de Curitiba, 782 do Recife, e 447 do Rio de Janeiro. Na Tabela 2 podem observar-se características dos participantes.

Tabela 2 - Caracterização dos participantes

\begin{tabular}{llccccc}
\hline & & $\begin{array}{c}\text { Belo Horizonte } \\
(\mathbf{n}=663)\end{array}$ & $\begin{array}{c}\text { Brasília } \\
(\mathbf{n}=6 \mathbf{6 2 6})\end{array}$ & $\begin{array}{c}\text { Curitiba } \\
(\mathbf{n}=5 \mathbf{2 1})\end{array}$ & $\begin{array}{c}\text { Recife } \\
(\mathbf{n}=777)\end{array}$ & $\begin{array}{c}\text { Rio de Janeiro } \\
(\mathbf{n}=446)\end{array}$ \\
\hline Gênero & Feminino & $32,1 \%$ & $32,5 \%$ & $37,6 \%$ & $28,1 \%$ & $33,8 \%$ \\
& Masculino & $67,9 \%$ & $67,5 \%$ & $62,4 \%$ & $71,9 \%$ & $66,2 \%$ \\
Idade (M) & & 32,7 & 32,7 & 32,7 & 30,8 & 35,1 \\
& Ensino básico & $25,9 \%$ & $26,8 \%$ & $19,2 \%$ & $24,5 \%$ & $14,1 \%$ \\
Formação & Ensino superior & $74,1 \%$ & $73,2 \%$ & $80,5 \%$ & $75,5 \%$ & $85,9 \%$ \\
Comprou & Sim & $88,8 \%$ & $91,1 \%$ & $86 \%$ & $84 \%$ & $89,5 \%$ \\
ingresso? & Não & $11,2 \%$ & $8,7 \%$ & $14 \%$ & $16 \%$ & $10,5 \%$ \\
Vai ao & Sim & $72,1 \%$ & $62,1 \%$ & $60,8 \%$ & $47,5 \%$ & $79,1 \%$ \\
futebol? & Não & $27,9 \%$ & $37,9 \%$ & $39,2 \%$ & $52,5 \%$ & $20,9 \%$ \\
Apoia a Copa & Sim & $83,1 \%$ & $87,5 \%$ & $78,7 \%$ & $72,5 \%$ & $86,3 \%$ \\
no Brasil? & Não & $16,9 \%$ & $12,5 \%$ & $21,3 \%$ & $27,5 \%$ & $13,7 \%$ \\
\hline
\end{tabular}

Fonte: Autoria própria, 2015.

\section{ANÁLISE DOS DADOS}

Foi realizada uma análise fatorial confirmatória (AFC) utilizando o AMOS 22.0, de forma a avaliar o ajustamento do modelo de medida. A fiabilidade composita (FC) foi estimada para avaliar a consistência interna dos fatores (HAIR et al., 2009), consideraram-se valores iguais ou superiores a 0,60 como indicativos de boa consistência interna (BAGGOZI; YI, 1988). A variância média extraída (VME) foi calculada para estimar a validade convergente, considerou-se o ponto de corte de 0,50 como indicador da validade convergente (FORNELL; LARCKER, 1981). Para a validade discriminante considerou-se o critério do valor das VME dos fatores serem superiores ou iguais ao quadrado das correlações entre estes fatores (FORNELL; LARCKER, 1981). A adequação do modelo de medida foi avaliada através do rácio do qui-quadrado $\left(\chi^{2}\right)$ para seus graus de liberdade, Comparative-of-Fit-Index (CFI), Good-of-Fit-Index (GFI), Tucker-Lewis index (TLI) e Root Mean Square Error of Aproximation (RMSEA). Em seguida, utilizou-se o SPSS 22.0 para realizar testes MANOVA com o objetivo de comparar a percepção da qualidade do evento entre os torcedores das cinco cidades-sede. Os pressupostos de normalidade multivariada foram estimados através do teste de Kolmogorov-Smirnov. Esta condição não foi assumida para nenhuma das cinco amostras de participantes $(p<0,05)$. No entanto, o tamanho da amostra para as cinco cidades-sede e os valores de assimetria e curtose menores do que $|3,0|$ e $|7,0|$, respectivamente, sugerem que as medidas poderiam ser utilizadas nas análises subsequentes (KLINE, 2005). Para avaliar a homogeneidade das variâncias foi utilizado o teste de Levene, não sendo esta condição assumida $(p<0,05)$. Usou-se depois o fator de correção de Welsh (MARÔCO, 2010), sendo a significância estatística entre os grupos assumida para $p<0,05$. Adicionalmente foram usados os testes post-hoc HSD de Tukey para verificar onde se encontravam as diferenças entre os cinco grupos (MARÔCO, 2010). 


\section{RESULTADOS}

\section{1 modelo de medida}

Inicialmente, o modelo de medida foi analisado para toda a amostra. Os resultados da AFC mostraram que o peso fatorial de dois itens não ultrapassaram 0,50 , sendo eliminados (HAIR et al., 2009). Adicionalmente, o quadrado da correlação entre os fatores acessibilidades e segurança foi superior ao valor da VME desses fatores, sugerindo falta de validade discriminante. Assim, os itens foram agrupados num fator único denominado acessibilidades, tendo em conta o conteúdo dos itens e a literatura (BISCAIA et al., 2013, YOSHIDA et al., 2013). O mesmo ocorreu com os fatores arena e atmosfera do evento, sendo os itens agrupados num fator denominado atmosfera do evento (YOSHIDA; JAMES; CRONIN, 2013). Em seguida, foi realizado um refinamento da escala e apenas os itens com maior peso fatorial em cada atributo foram selecionados de modo a assegurar a fiabilidade e parcimónia do modelo (GLADDEN; FUNK, 2002). O modelo de medida final foi composto por 16 itens (dois nas dimensões equipes e atmosfera do evento; três nas dimensões árbitros, pessoal de apoio, acessibilidades e experiência com os torcedores).

Após o refinamento, o modelo de medida foi testado para cada uma das cidades-sede (Tabela 3). Os valores da fiabilidade compósita foram próximos ou superiores a 0,60 (BAGGOZI; YI, 1988), indicando consistência interna aceitável. Todos os valores da VME foram próximos ou superiores a 0,50 (FORNELL; LARCKER, 1981). Além disso, o valor médio de VME para cada cidade-sede foi superior a 0,50 (BH=0,55; $B S B=0,52$; $C W B=0,51 ; R E C=0,55 ; \mathrm{RIJ}=0,52$ ). Adicionalmente, os resultados do modelo final de medida mostraram um ajuste aceitável para as cidades-sede (Tabela 3). 0 valor $\chi^{2}$ foi significativo para todos os grupos e o seu rácio para os graus de liberdade foi acima do critério 3,0 (KLINE, 2005) apenas para o modelo com participantes do Recife (Tabela 3). Não obstante, é importante considerar outros índices uma vez que este estudo foi realizado com amostras de grande dimensão e a estatística $\chi^{2}$ tende a ser sensível ao tamanho da amostra (HAIR et al., 2009). Os valores TLI, CFI, GFI foram superiores ao limite recomendado de 0,90 (HAIR et al., 2009). Adicionalmente, o RMSEA foi inferior ou igual a 0,06 em todos os modelos, fornecendo evidências de bom ajuste. Em geral, o modelo de medida para cada cidade-sede mostrou um ajuste aceitável.

Seguidamente, os resultados dos testes MANOVA indicaram diferenças significativas (Lambda de Wilks= 0,834, $p<0,001$ ) entre os participantes das cinco cidades-sede para todos os atributos da qualidade do evento (Tabela 4).

Tabela 3 - Resultados da Análise Fatorial Confirmatória

\begin{tabular}{|c|c|c|c|c|c|c|c|c|c|}
\hline Atributos & Peso Fatorial & $\mathrm{FC}$ & VME & 1 & 2 & 3 & 4 & 5 & 6 \\
\hline \multicolumn{10}{|l|}{ Belo Horizonte $(n=663)$} \\
\hline \multicolumn{10}{|c|}{ Model fit: $\chi^{2}(89)=258,618(p<.001), \chi^{2} / \mathrm{df}=2,90, \mathrm{TLI}=0,945, \mathrm{CFI}=0,959, \mathrm{GFI}=0,952, \mathrm{RMSEA}=0,05$} \\
\hline 1. Equipes & $0,64-0,65$ & 0,58 & 0,41 & 1,00 & & & & & \\
\hline 2. Árbitros & $0,64-0,74$ & 0,76 & 0,51 & 0,32 & 1,00 & & & & \\
\hline 3. Pessoal de apoio & $0,75-0,83$ & 0,84 & 0,64 & 0,33 & 0,28 & 1,00 & & & \\
\hline 4. Acessibilidades & $0,69-0,72$ & 0,75 & 0,66 & 0,32 & 0,27 & 0,49 & 1,00 & & \\
\hline 5. Atmosfera do evento & $0,73-0,88$ & 0,80 & 0,50 & 0,21 & 0,07 & 0,36 & 0,22 & 1,00 & \\
\hline 6. Experiência com os torcedores & $0,69-0,81$ & 0,82 & 0,60 & 0,38 & 0,10 & 0,31 & 0,29 & 0,33 & 1,00 \\
\hline
\end{tabular}


Continuação da tabela $3 .$.

\begin{tabular}{|c|c|c|c|c|c|c|c|c|c|}
\hline \multicolumn{10}{|l|}{ Brasília ( $n=626)$} \\
\hline \multicolumn{10}{|c|}{ Model fit: $\chi^{2}(89)=194,294(p<.001), \chi^{2} / \mathrm{df}=2,18, \mathrm{TLI}=0,958, \mathrm{CFI}=0,969, \mathrm{GFI}=0,964, \mathrm{RMSEA}=0,04$} \\
\hline 1. Equipes & $0,65-0,69$ & 0,62 & 0,45 & 1,00 & & & & & \\
\hline 2. Árbitros & $0,68-0,83$ & 0,79 & 0,56 & 0,19 & 1,00 & & & & \\
\hline 3. Pessoal de apoio & $0,73-0,80$ & 0,82 & 0,60 & 0,23 & 0,22 & 1,00 & & & \\
\hline 4. Acessibilidades & $0,60-0,72$ & 0,71 & 0,44 & 0,27 & 0,13 & 0,52 & 1,00 & & \\
\hline 5. Atmosfera do evento & $0,67-0,73$ & 0,66 & 0,49 & 0,26 & 0,25 & 0,43 & 0,41 & 1,00 & \\
\hline 6. Experiência com os torcedores & $0,66-0,77$ & 0,78 & 0,55 & 0,23 & 0,01 & 0,22 & 0,38 & 0,32 & 1,00 \\
\hline \multicolumn{10}{|l|}{ Curitiba $(n=523)$} \\
\hline \multicolumn{10}{|c|}{ Model fit: $\chi^{2}(89)=255,231(p<.001), \chi^{2} / \mathrm{df}=2,86, \mathrm{TLI}=0,918, \mathrm{CFI}=0,939, \mathrm{GFI}=0,942, \mathrm{RMSEA}=0,06$} \\
\hline 1. Equipes & $0,65-0,73$ & 0,65 & 0,48 & 1,00 & & & & & \\
\hline 2. Árbitros & $0,66-0,77$ & 0,76 & 0,51 & 0,43 & 1,00 & & & & \\
\hline 3. Pessoal de apoio & $0,66-0,82$ & 0,78 & 0,54 & 0,25 & 0,16 & 1,00 & & & \\
\hline 4. Acessibilidades & $0,60-0,71$ & 0,71 & 0,45 & 0,24 & 0,18 & 0,45 & 1,00 & & \\
\hline 5. Atmosfera do evento & $0,78-0,83$ & 0,78 & 0,64 & 0,25 & 0,20 & 0,34 & 0,25 & 1,00 & \\
\hline 6. Experiência com os torcedores & $0,64-0,70$ & 0,71 & 0,45 & 0,18 & 0,11 & 0,31 & 0,48 & 0,37 & 1,00 \\
\hline
\end{tabular}

\section{Recife ( $\mathrm{n}=781)$}

Model fit: $\chi^{2}(89)=284,492(p<.001), \chi^{2} / \mathrm{df}=3,19, \mathrm{TLI}=0,945, \mathrm{CFI}=0,959, \mathrm{GFI}=0,956, \mathrm{RMSEA}=0,05$

\begin{tabular}{llllllllll} 
1. Equipes & $0,60-0,70$ & 0,60 & 0,43 & 1,00 & & & & & \\
2. Árbitros & $0,68-0,77$ & 0,77 & 0,52 & 0,44 & 1,00 & & & & \\
3. Pessoal de apoio & $0,73-0,81$ & 0,83 & 0,62 & 0,38 & 0,41 & 1,00 & & & \\
4. Acessibilidades & $0,65-0,70$ & 0,73 & 0,48 & 0,25 & 0,35 & 0,45 & 1,00 & & \\
5. Atmosfera do evento & $0,68-0,77$ & 0,69 & 0,53 & 0,43 & 0,27 & 0,51 & 0,42 & 1,00 & \\
6. Experiência com os torcedores & $0,70-0,81$ & 0,81 & 0,59 & 0,44 & 0,10 & 0,25 & 0,22 & 0,43 & 1,00 \\
\hline
\end{tabular}

\section{Rio de Janeiro $(\mathrm{n}=449)$}

Model fit: $\chi^{2}(89)=204,745(p<.001), \chi^{2} / d f=2,30, T L I=0,931, C F I=0,949, G F I=0,948, R M S E A=0,05$

\begin{tabular}{llllllllll} 
1. Equipes & $0,64-0,68$ & 0,61 & 0,44 & 1,00 & & & & & \\
2. Árbitros & $0,62-0,71$ & 0,74 & 0,48 & 0,24 & 1,00 & & & & \\
3. Pessoal de apoio & $0,69-0,85$ & 0,80 & 0,57 & 0,35 & 0,12 & 1,00 & & & \\
4. Acessibilidades & $0,65-0,74$ & 0,74 & 0,49 & 0,22 & 0,11 & 0,45 & 1,00 & & \\
5. Atmosfera do evento & $0,65-0,83$ & 0,71 & 0,55 & 0,26 & 0,06 & 0,40 & 0,37 & 1,00 & \\
6. Experiência com os torcedores & $0,60-0,80$ & 0,75 & 0,51 & 0,30 & 0,05 & 0,20 & 0,18 & 0,31 & 1,00 \\
\hline
\end{tabular}

Fonte: Autoria própria.

$\mathrm{Na}$ Tabela 4, são apresentados os resultados dos testes da MANOVA para comparação dos atributos da qualidade entre as diferentes cidades. No atributo equipes ( $F=66,16, p=0,001)$, com exceção de Brasília na comparação com Rio de Janeiro, verificaram-se diferenças significativas entre todas as cidades. Curitiba apresentou a maior média $(M=6,02, D P=0,91)$, enquanto Recife teve a menor média ( $M=5,18, D P=1,16)$. No atributo árbitros ( $F=6,26, p=0,001)$ diferenciaram-se Belo Horizonte em relação ao Rio de Janeiro, e Curitiba em relação à Belo Horizonte, a Brasília e ao Recife. Nesta dimensão, os resultados de Curitiba apresentaram maior valor de média $(\mathrm{M}=4,83, \mathrm{DP}=1,04)$, sendo o menor valor observado em Belo Horizonte $(M=4,54, D P=1,08)$. No atributo pessoal de apoio $(F=38,49, p=0,001)$ não foram encontradas diferenças significativas na comparação entre o Rio de Janeiro em relação a Brasília e a Curitiba. Também neste atributo, Curitiba manteve o maior valor de média $(M=5,43, D P=1,03) \mathrm{e}$ Recife o menor valor $(M=4,76, D P=1,22)$. No atributo acessibilidades ( $F=98,55, p=0,001)$, com 
exceção da comparação entre Brasília e Rio de Janeiro, todas as cidades-sede apresentaram diferenças significativas. A maior e a menor média neste atributo foram observadas em Curitiba $(M=5,80, D P=0,99)$ e Recife $(M=4,64, D P=1,24)$ respectivamente. No atributo atmosfera do evento $(F=20,56, p=0,001)$ não foram observadas diferenças entre Curitiba em relação a Belo Horizonte e a Brasília, e Brasília em relação ao Rio de Janeiro. Ainda sobre a atmosfera do evento, o Rio de Janeiro obteve o maior valor médio $(M=5,65$, $D P=1,35)$ e o Recife o menor $(M=4,98, D P=1,38)$. No atributo experiência com o torcedor $(F=42,31, p=0,001)$, não foram encontradas diferenças significativas entre Belo Horizonte e Brasília. O mesmo sucedeu para Curitiba em relação a Brasília e Rio de Janeiro. Todas as outras comparações apresentaram diferenças significativas. Neste atributo, o Rio de Janeiro obteve o maior valor médio $(M=6,38$, $\mathrm{DP}=0,7)$ e Recife o menor $(\mathrm{M}=5,72, \mathrm{DP}=1,18)$.

Tabela 4 - Resultados dos testes da MANOVA

\begin{tabular}{|c|c|c|c|c|c|c|}
\hline & \multicolumn{6}{|c|}{ Atributos } \\
\hline & Equipes & Árbitros & $\begin{array}{c}\text { Pessoal de } \\
\text { apoio }\end{array}$ & $\begin{array}{l}\text { Acessibi- } \\
\text { lidades }\end{array}$ & $\begin{array}{l}\text { Atmosfera } \\
\text { do evento }\end{array}$ & $\begin{array}{c}\text { Experiência com } \\
\text { os torcedores }\end{array}$ \\
\hline $\mathbf{F}$ & 66,16 & 6,26 & 38,49 & 98,55 & 20,56 & 42,31 \\
\hline Sig. & 0,001 & 0,001 & 0,001 & 0,001 & 0,001 & 0,001 \\
\hline Potencia do teste & 1,00 & 0,98 & 1,00 & 1,00 & 1,00 & 1,00 \\
\hline Cidades & $M(D P)$ & $M(D P)$ & $M(D P)$ & $M(D P)$ & $M(D P)$ & $M(D P)$ \\
\hline Belo Horizonte (BHZ) & $5,44(1,04)$ & $4,54(1,08)$ & $4,95(1,14)$ & $4,99(1,11)$ & $5,20(1,43)$ & $6,11(0,99)$ \\
\hline Brasília (BSB) & $5,69(0,94)$ & $4,60(1,24)$ & $5,23(1,03)$ & $5,41(1,00)$ & $5,43(1,18)$ & $6,16(0,88)$ \\
\hline Curitiba (CWB) & $6,02(0,91)$ & $4,83(1,04)$ & $5,43(1,03)$ & $5,80(0,99)$ & $5,30(1,37)$ & $6,29(0,85)$ \\
\hline Recife (REC) & $5,18(1,16)$ & $4,64(1,22)$ & $4,76(1,22)$ & $4,64(1,24)$ & $4,98(1,38)$ & $5,72(1,18)$ \\
\hline \multirow[t]{2}{*}{ Rio de Janeiro (RIO) } & $5,80(0,91)$ & $4,77(1,17)$ & $5,33(1,14)$ & $5,31(1,15)$ & $5,65(1,35)$ & $6,38(0,77)$ \\
\hline & \multicolumn{6}{|c|}{ Comparação dos atributos da qualidade entre as cidades-sede } \\
\hline $\mathrm{BHZ} \times \mathrm{BSB}$ & 0,001 & 0,877 & 0,001 & 0,001 & 0,022 & 0,866 \\
\hline $\mathrm{BHZ} \times \mathrm{CWB}$ & 0,001 & 0,001 & 0,001 & 0,001 & 0,744 & 0,018 \\
\hline $\mathrm{BHZ} \times \mathrm{REC}$ & 0,001 & 0,457 & 0,013 & 0,001 & 0,016 & 0,001 \\
\hline $\mathrm{BHZ} \times \mathrm{RIO}$ & 0,001 & 0,008 & 0,001 & 0,001 & 0,001 & 0,001 \\
\hline BSB $\times$ CWB & 0,001 & 0,006 & 0,023 & 0,001 & 0,480 & 0,214 \\
\hline BSB $\times$ REC & 0,001 & 0,966 & 0,001 & 0,001 & 0,001 & 0,001 \\
\hline BSB x RIO & 0,367 & 0,111 & 0,580 & 0,664 & 0,055 & 0,003 \\
\hline CWB $\times$ REC & 0,001 & 0,028 & 0,001 & 0,001 & 0,001 & 0,001 \\
\hline CWB x RIO & 0,009 & 0,931 & 0,665 & 0,001 & 0,001 & 0,559 \\
\hline REC x RIO & 0,001 & 0,301 & 0,001 & 0,001 & 0,001 & 0,001 \\
\hline
\end{tabular}

Fonte: Autoria própria.

\section{DISCUSSÃO}

O objetivo deste estudo foi avaliar a percepção da qualidade da Copa do Mundo da FIFA Brasil 2014 entre os torcedores de diferentes cidades-sede. Esta pesquisa presta um contributo para a literatura sobre eventos esportivos na medida em que: (1) examina se a percepção de qualidade do evento varia significativamente entre torcedores em diferentes cidades, e (2) lança um olhar sobre a importância do contexto (i.e., cultural, social e econômico) na análise da qualidade dos megaeventos esportivos. 
A estrutura fatorial obtida indicou boas qualidades psicométricas, permitindo indicar que o modelo é adequado para avaliar a qualidade deste megaevento esportivo. Adicionalmente, importa referir que a caracterização da amostra apresentada na Tabela 2 sugere que os participantes das cinco cidades-sede têm um perfil consistente ao que foi descrito pela Fundação Getulio Vargas (2014) sobre a Copa do Mundo (i.e., 53\% dos espectadores nas doze cidades-sede possuíam no mínimo a graduação, 43\% frequentam os estádios de futebol no país e $95,1 \%$ foram aos jogos como espectadores com bilhetes comprados).

Os resultados dos testes MANOVA indicam que a percepção da qualidade do megaevento foi avaliada de forma significativamente diferente entre os torcedores nas cidadessede. Esses resultados estão em linha com estudos que sugerem que a percepção dos consumidores sobre a qualidade do serviço tende a variar consonante o contexto onde se faz a avaliação (BISCAIA et al., 2013, UETSCHY; KRAMFT, 2000). Ou seja, diferentes contextos culturais, económicos e sociais podem impactar na percepção da qualidade do evento. Assim, é crucial que os anfitriões controlem e monitorem os atributos do evento que estejam ao seu alcance (MURRAY; HOWAT, 2002).

$\mathrm{Na}$ comparação dos atributos da qualidade do evento entreas cinco cidades-sede, as diferenças observadas sugerem que os torcedores com maior nível de formação (i.e., Curitiba e Rio Janeiro; Tabela 2) avaliam de forma mais positiva a qualidade do evento (Tabela 4). Por outro lado, a cidade onde a qualidade foi avaliada de forma menos positiva foi Recife. Essa cidade apresenta o menor número de torcedores a favor da realização do evento no país, sugerindo que quem não apoia o evento tende a ter uma percepção menos favorável dos atributos da qualidade do serviço prestado (BISCAIA et al., 2015). Além disso, Recife é a cidade com menor número de participantes que frequentam o futebol, confirmando a ideia de que as experiências anteriores em eventos esportivos influenciam a percepção em relação à qualidade do evento (HSIEH; WANG; YODER, 2011). Nota-se também que os indicadores de desenvolvimento apresentados das cidades podem ajudar a compreender as diferenças observadas. Por exemplo, Curitiba é a cidade com melhor qualidade de vida e menor nível de pobreza, enquanto Recife tem menor rendimento, maior nível de pobreza e menor qualidade de vida (Tabela 1). Esses dados estão em linha com a ideia de que a percepção da qualidade dos eventos pode diferir de acordo com a qualidade de vida (KIM; YOO; PEDERSON, 2007). Deste modo, é imperativo, por parte dos anfitriões, avaliar previamente que implicações terá o evento do ponto de vista social, económico e estrutural nos locais de acolhimento. Tal avaliação deverá servir para que os valores investidos na realização do evento possam ter impacto positivo para os residentes (RODRIGUES SOARES, 2013).

Numa avaliação pontual das cidades onde a diferença na percepção dos atributos da qualidade foi mais notória (Belo Horizonte, Curitiba, Recife e Rio de Janeiro), destacamse as acessibilidades e o pessoal de apoio. $O$ fato de os torcedores do Recife avaliarem de forma menos positiva as acessibilidades do que os das outras três cidades pode estar ligado aos constrangimentos inerentes ao deslocamento (i.e., distância, transporte, clima, tempo de deslocamento) para a arena (TORCEDORES..., 2013,VEJA..., 2014). Isto porque, em Recife, uma nova arena foi construída a cerca de 20 quilómetros do centro da cidade com o objetivo de criar uma nova centralidade e reestruturar todo o setor oeste da Região Metropolitana (RAMALHO; VASCONCELOS, 2012). Já em Belo Horizonte, Curitiba e Rio de Janeiro as arenas são estruturas reformadas já conhecidas e frequentadas pelos torcedores do futebol, e a sua 
localização fica próxima ao centro das respectivas cidades. Esses resultados podem ajudar a entender o impacto da reorganização urbana proporcionada pelos megaeventos. Ou seja, nas cidades onde as arenas foram construídas especialmente para o evento (e.g., Recife), os impactos urbanos podem gerar diferenças na percepção da qualidade (RODRIGUES SOARES, 2013). Neste sentido, garantir a rapidez e a segurança nos acessos através de efetivos sistemas de transportes pode ajudar a melhorar a percepção das acessibilidades. Adicionalmente, a percepção menos positiva sobre o pessoal de apoio no Recife em comparação com Belo Horizonte, Curitiba e Rio de Janeiro pode estar associada à sensação de menor segurança no deslocamento para a arena (MOTORISTA..., 2014). Pode-se referir ainda que têm ocorrido alguns episódios de violência em estádios no Brasil (RASPAUD; BASTOS, 2013). Deste modo, ações conjuntas dos anfitriões e da polícia para auxiliar os torcedores, reforçar a segurança e controlar os incidentes durante o período em que o evento decorre podem evitar efeitos negativos na percepção da qualidade do megaevento.

Os resultados também apontam que entre o Rio de Janeiro e Brasília não se encontram diferenças significativas na percepção sobre a qualidade do evento (Tabela 4). Isto pode sugerir que entre estas cidades existem semelhanças que contribuem para a inexistência de diferenças na percepção (KIM; YOO; PEDERSON, 2007). Por exemplo, estas foram as cidades onde 0 apoio à realização da Copa foi mais significativo e onde houve um maior número de torcedores que compraram ingressos (Tabela 2). No plano político, social e econômico, ambas centralizam boa parte das estruturas administrativas dos órgãos estatais. Brasília, por ser a capital e 0 Rio de Janeiro com a herança do período em que foi capital (INSTITUTO BRASILEIRO DE GEOGRAFIA E ESTATÍSTICA, 2014). Economicamente, estas duas cidades têm o maior PIB e maior participação no PIB nacional entre as cinco pesquisadas, sendo que os investimentos na Copa foram também os maiores (Tabela 1).

\section{CONCLUSÕES, LIMITAÇÕES E SUGESTÕES DE PESQUISA}

Concluindo, esse estudo representa um passo importante para fornecer informações relativas à percepção da qualidade dos megaeventos por parte dos torcedores no contexto do futebol devido à natureza da abordagem. Como objetivo, buscou fornecer indicações sobre a influência do contexto social, econômico e cultural na percepção da qualidade do evento por parte dos torcedores.

Os resultados sugerem que quanto mais elevado é o nível de formação dos torcedores e a qualidade de vida no local da realização do evento, maior tende a ser a avaliação da qualidade do evento. Por outro lado, os resultados também indicam que a pior avaliação da qualidade da Copa do Mundo FIFA Brasil 2014 aconteceu nas cidades que apresentam menores níveis de desenvolvimento e menor índice de aprovação para a realização do evento. É de notar também que, nas cidades onde foram revitalizadas infraestruturas para a realização do evento, a qualidade foi superiormente avaliada quando comparada às cidades onde foram necessários investimentos para construção de novas infraestruturas para acolher os jogos da competição.

Desse modo, somos de opinião que é imperativo, por parte dos anfitriões, salientar os impactos sociais advindos do evento para a comunidade, e garantir que a transformação urbana e a reorganização da estrutura social tenham impacto positivo na vida dos residentes. 
No que diz respeito aos atributos da qualidade utilizados neste estudo, os resultados apontam para as dimensões: pessoal de apoio, segurança e acessibilidades como as mais importantes para os torcedores na Copa do Mundo FIFA Brasil 2014. Isto permite-nos concluir que é fundamental que os organizadores garantam que as experiências no evento possam ser positivas e previamente planejadas e monitoradas, a fim de assegurar uma melhor avaliação sobre os atributos da qualidade do evento.

Para finalizar, é importante referir que este estudo apresenta limitações que devem ser consideradas em futuras pesquisas. Primeiro, os dados foram coletados em apenas cinco das 12 cidades-sede. Os resultados podem não ser representativos de todos os torcedores da Copa. Estudos adicionais sobre megaeventos esportivos devem recolher dados em todas as subsedes. Além disso, convém relembrar que os dados foram coletados antes de os torcedores entrarem nas arenas. Deste modo, ainda que o evento comece muito antes do jogo, será importante no futuro coletar dados antes, durante e depois dos jogos, a fim de se compreender como as percepções dos torcedores variam de acordo com o quadro temporal. Segundo, a maioria dos participantes tinha graduação ou pós-graduação e a maioria indicou comprar seus ingressos para o evento. Este aspeto pode sugerir que a amostra apresenta características que não se adequam a toda a população brasileira, pelo que em futuros estudos será importante recolher amostras representativas da população do país anfitrião. De referir também que não foi recolhida informação sobre os gastos totais com o evento (i.e., bilhete, comida e bebida, transportes). Em estudos futuros, a inclusão de variáveis relativas ao investimento financeiro para assistir ao evento pode ser importante para melhor compreender os comportamentos de consumo e a avaliação da qualidade do evento (RITCHIE; SHIPWAY; CLEEVE, 2009, SNELGROVE et al., 2008).

\section{REFERÊNCIAS}

AGARWAL, James; MALHOTRA, Naresh; BOLTON, Ruth. A cross-national and cross-cultural approach to global market segmentation: an application using consumers perceived service quality. Journal of International Marketing, Chicago, v. 18, n. 3, p 18-40, 2010.

AU, Kevin. Intra-cultural variation: evidence and implications for international business. Journal of International Business Studies, Michigan, v. 30, n 4, p. 799-812, 1999.

BABIN, Barry; ATTAWAY, Jill. Atmospheric affect as a tool for creating value and gaining share of customer. Journal of Business Research, Oxford, v. 49, n 2, p. 91-99, 2000.

BAGOZZI, Richard; YI, Youjae. On the evaluation of structural equation models. Journal of the Academy of Marketing Science, Dordrecht, v. 16, n. 1, p. 74-94, 1988.

BISCAIA Rui et al. The 2014 FIFA World Cup: examining service quality and value perceptions among attendees at Rio de Janeiro. In: North American Society for Sports Management, 2015. Conference Abstract of the North American Society for Sport Management. [Anais...] Ottawa, NASSM, 2015. 210. Disponível em:<https://www.nassm.com/ files/conf abstracts/2015-014.pdf>. Acesso em: 20 mar. 2015.

BISCAIA, Rui et al. The role of service quality and ticket price on satisfaction and behavioral intentions within the professional soccer context. International Journal of Sports Marketing \& Sponsorship, Bingley, v. 14, n. 4, p. 301-325, 2013.

BRINGING FIFA to Brazil equal to roughly $61 \%$ of education budget. 2014. Disponivel em: <http:// www.forbes.com/sites/kenrapoza/2014/06/11/bringing-fifa-to-brazil-equal-to-roughly-61-of- 
education-budget/>. Acesso em: 20 mar. 2015.

CAETANO, Raul; PINSKY, llana; LARANJEIRA, Ronaldo. Should soccer and alcohol mix? Alcohol sales during the World Soccer Cup Games in Brazil. Addiction, Abingdon, v. 107, p. 1722-1723, 2012.

$\mathrm{CHAO}$, Wen et al. The role of national culture on relationships between customers perception of quality, values, satisfaction, and behavioral intentions. Quality Management Journal, Milwaukee, v. 19, n. 4, p. 7-23, 2012.

CRONIN, Joseph; BRADY, Michael; HULT, Tomas. Assessing the effects of quality, value and customer satisfaction on consumer behavioral intentions in service environments. Journal of Retailing, Oxford, v. 76, n. 2, p. 193-218, 2000.

DEMBEK, Agata; WŁOCH, Renata. The impact of a sports mega-event on the international image of a country: the case of Poland hosting UEFA Euro 2012. Perspectives: Central European Review of International Affairs, Malá Strana, v. 22, n. 1, p. 33-47, 2014.

ESPN Schedule for 2014 FIFA World Cup. 2014. Disponível em: <http://espnmediazone.com/us/pressreleases/2014/02/espn-schedule-2014-fifa-world-cup/>. Acesso em: 11 jan. 2015

\section{FÉDÉRATION INTERNATIONALE DE FOOTBALL ASSOCIATION. FIFA fan fest locations}

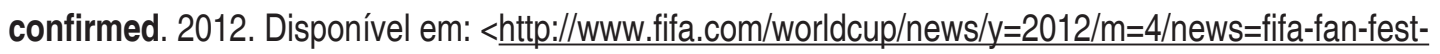
locations-confirmed-1614471.html >. Acesso em: 11 jan. 2015.

FÉDÉRATION INTERNATIONALE DE FOOTBALL ASSOCIATION. Russia launches major Infrastructure Programme for 2018. 2013. Disponível em: <http://www.fifa.com/worldcup/news/ $\mathrm{y}=2013 / \mathrm{m}=6 /$ news=russia-launches-major-infrastructure-programme-for-2018-2111299.html. $>$. Acesso em: 31 mar. 2015.

FLOREK, Magdalena; BREITBARTH, Tim; CONEJO, Francisco. Mega Event=Mega Impact? Travelling fans experience and perceptions of the 2006 FIFA World Cup Host Nation. Journal of Sport \& Tourism, Oxfordshire, v. 13, n. 3, p. 199-219, 2008.

FORNELL, Claes; LARCKER, David. Evaluating structural equation models with unobservable variables and measurement error. Journal of Marketing Research, Chicago, v. 18, n. 1, p. 39-50, 1981.

FUNDAÇÃO GETULIO VARGAS. Pesquisa de demanda turística doméstica na Copa do Mundo da FIFA no Brasil 2014. 2014. Disponível em: <http://www.dadosefatos.turismo.gov.br/export/sites/ default/dadosefatos/outros_estudos/downloads_outrosestudos/Estudos_da_Demanda_Domestica Brasil Copa 2014 1.pDP>Acesso em: 31 mar. 2015.

GLADDEN, James; FUNK, Daniel. Developing an understanding of brand associations in team sport: empirical evidence from consumers of professional sport. Journal of Sport Management, Champaing, v. 16, n. 1, p. 54, 2002.

HAIR, Joseph et al. Multivariate data analyses. 7· ed. New York: Prentice Hall, 2009.

HEERE, Bob et al. The power of sport to unite a nation: the social value of the 2010 FIFA World Cup in South Africa. European Sport Management Quarterly, Oxfordshire, v. 13, n. 4, p. 450-471, 2013.

HSIEH, Li-Wen; WANG, Chien-Hsin; YODER, Tracy. Factors Associated with Professional Baseball Consumption: a cross-cultural comparison study. International Journal of Business \& Information, Taipei, v. 6, n. 2, p. 135-159, 2011.

INOUE, Yuhei; HAVARD, Cody. Determinants and consequences of the perceived social impact of a sport event. Journal of Sport Management, Champaing, v. 28, n. 3, p. 295-310, 2014.

INSTITUTO BRASILEIRO DE GEOGRAFIA E ESTATÍSTICA. Produto Interno Bruto dos Municipios 2010. 2010. Disponível em: <ttp://ttp.ibge.gov.br/Pib Municipios/2010/pibmunic2010. pDP>. Acesso em: 2 mar. 2015. 
INSTITUTO BRASILEIRO DE GEOGRAFIA E ESTATÍSTICA. São Paulo, Brasília e Rio de Janeiro centralizam principais redes de gestão do território nacional. 2014. Disponível em: $<$ http://saladeimprensa.ibge.gov.br/noticias?idnoticia=2622\&view=noticia $>$. Acesso em: $19 \mathrm{abr}$. 2015.

JEEYOON, Kim; JOON HO, Kang; YU-KYOUM, Kim. Impact of Mega Sport Events on Destination Image and Country Image. Sport Marketing Quarterly, Morgantown, v. 23, n. 3, p. 161-175, 2014.

KAPLANIDOU, Kyriaki. The importance of legacy outcomes for Olympic Games four summer host cities residents quality of life: 1996-2008. European Sport Management Quarterly, Oxfordshire, v. 12, p.397-433, 2012.

KIM, Sangho; YOO, Euidong.; PEDERSON, Paul. Market segmentation in the K-League: an analysis of spectators of the Korean professional soccer league. International Journal of Sports Marketing and Sponsorship, Bingley, v. 8, n. 2, p. 141-158, 2007.

KLINE, Rex. Principles and practice of structural equation modeling. 2. ed. New York, NY: The Guilford, 2005.

KOENIGSTORFER, Joerg; GROEPPEL-KLEIN, Andrea; KUNKEL, Thilo. The attractiveness of national and international football leagues: perspectives of fans of "star clubs" and "underdogs". European Sport Management Quarterly, Oxfordshire, v. 10, n. 2, p. 127-163, 2010.

$\mathrm{KOO}$, Gi-Yong et al. Examination of the casual effects between the dimensions of service quality and spectator satisfaction in minor league baseball. International Journal of Sports Marketing \& Sponsorship, Bingley, v. 11, n. 1, p. 46-59, 2009.

LIU, Yi-De; TAYLOR, Peter; SHIBLI, Simon. Measuring customer service quality of English public sport facilities. International Journal of Sport Management and Marketing, Buckinghamshire, v. 6, n. 3, p. 229-252, 2009.

MARÔCO, João. Análise estatística com o SPSS. 5.ed. [s.I.]:Pêro Pinheiro, 2010.

MOTORISTA enfrenta buracos e falta de iluminação para chegar até Arena PE. 2014. Disponível em: $<$ http://g1.globo.com/pernambuco/noticia/2014/05/motorista-enfrenta-buracos-e-falta-de-iluminacaopara-chegar-ate-arena-pe.html>. Acesso em: 16 mar. 2015.

MURRAY, Duncan; HOWAT, Gary. The relationship among service quality, value, satisfaction, and future intentions of customers at an Australian sports and leisure centre. Sport Management Review, Southport, v. 5, n. 1, p. 25-43, 2002.

PORTER, Philip; FLETCHER, Deborah. The economic impact of the Olympic Games: ex ante predictions and ex poste reliability. Journal of Sport Management, Champaing, v. 22, n. 4, p. 470486, 2008.

RAMALHO, Ana; VASCONCELOS, Ronald. A Copa do Mundo de 2014: impactos ou legados para as cidades-sede do Nordeste? In: ZIMERMAN, A. (Org.) . Copa do Mundo de 2014: impactos e legado. Santo André: Universidade Federal do ABC, 2013. v. 1.

RASPAUD, Michel; BASTOS, Flávia. Torcedores de futebol: Violence and public policies in Brazil before the 2014 FIFA World Cup. Sport in Society: Cultures, Commerce, Media, Politics, Oxfordshire, v. 16, n. 2, p. 192-204, 2013.

RITCHIE, Brent; SHIPWAY, Richard; CLEEVE, Bethany Resident perceptions of mega-sporting events: a non-host city perspective of the 2012 London Olympic games. Journal of Sport \& Tourism, Oxfordshire, v. 14, n. 2-3, p. 143-167, 2009.

RODRIGUES SOARES, Paulo. Megaeventos esportivos e o urbano: a Copa do Mundo de $2014 \mathrm{e}$ seus impactos nas cidades brasileiras. Revista FSA, Teresina, v. 10, n. 4, p. 195-214, 2013.

SANT, Stacy-Lynn; MASON, Daniel. Framing event legacy in a prospective host city: managing Vancouver's Olympic bid. Journal of Sport Management, Champaing, v. 29, n. 1, p. 42-56, 2015. 
SANTOS, João. The International Journal of the History of Sport, Oxfordshire, v. 31, n. 10, p. 1312-1327, 2014.

SNELGROVE, Ryan et al. How visitors and locals at a sport event differ in motives and identity. Journal of Sport \& Tourism, Oxfordshire, v. 13, n. 3, p. 165-180, 2008.

TAKS, Marijke; CHALIP, Laurence; GREEN, Christine. Impacts and strategic outcomes from nonmega sport events for local communities. European Sport Management Quarterly, Oxfordshire, v. 15, n. 1, p. 1-6, 2015.

THEODORAKIS, Nicholas et al. Predicting spectators behavioural intentions in professional football: The role of satisfaction and service quality. Sport Management Review, Southport, v. 16, n. 1, p. 8596, 2013.

THEODORAKIS, Nicholas; ALEXANDRIS, Kostantinos. Can service quality predict spectators behavioral intentions in Professional soccer? Managing Leisure, Oxfordshire, v. 13, n. 3-4, p. 162178, 2008.

TORCEDORES enfrentam maratona para chegar à Arena Pernambuco. 2013. Disponível em: <http:// www.bbc.co.uk/portuguese/noticias/2013/06/130617 copa recife cc bg> Acesso em: 15 mar. 2015.

UETSCHY, Linda; KRAMFT, Robert. Cultural sensitivity to satisfaction and service quality measures. Journal of Marketing Theory \& Practice, Philadelphia, v. 9, n. 3, p. 14-31, 2000.

VEJA o que não deu certo nas sedes da Copa durante 1ạ fase. 2014. Disponível em: <http://esportes. terra.com.br/futebol/copa-2014/veja-o-que-nao-deu-certo-nas-sedes-da-copa-durante-1-fase,c3a1d22 a935c6410VgnVCM3000009af154d0RCRD.html>. Acesso em: 15 mar. 2015.

WAKEFIELD, Kirk; BLODGETT, Jeffrey; SLOAN, Hugh. Measurement and management of the sportscape. Journal of Sport Management, Champaing, v. 10, n. 1, p. 15-31, 1996.

YOSHIDA, Masayuki; JAMES, Jeffrey. Service quality at sporting events: Is aesthetic quality a missing dimension? Sport Management Review, Oxford, v. 14, n. 1, p. 13-24, 2011.

YOSHIDA, Masayuki; JAMES, Jeffrey; CRONIN, Joseph. Value creation: assessing the relationships between quality, consumption value and behavioural intentions at sporting events. International Journal of Sports Marketing \& Sponsorship, Bingley, v. 14, n. 2, p. 126-148, 2013.

ZEITHAML, Valerie A.; BITNER, Mary. Services marketing: integrating customer focus across the firm. 3. ed. New York: McGraw Hill, 2003. 